\title{
MICROWAVE IMAGING OF MULTIPLE DIELECTRIC OBJECTS BY FDTD AND APSO
}

\author{
Chung-Hsin Huang, Chien-Hung Chen, Jau-Je Wu and Dar-Sun Liu \\ Department of Marine Engineering, \\ Taipei College of Maritime Technology, Taipei City, Taiwan, R.O.C. \\ havehuang@hotmail.com \\ f1092@mail.tcmt.edu.tw \\ darsunemail.tcmt.edu.tw \\ jaujewu@mail.tcmt.edu.tw
}

\begin{abstract}
An imaging approach to clear detection of two-dimensional geometries is proposed in this paper. The imaging reconstruction of multiple dielectric objects is retrieved by finite difference time domain (FDTD) method and the asynchronous particle swarm optimization (APSO) to determine the shape, location and permittivity of each dielectric object. The forward problem is solved based on the subgrid FDTD method by using EM pulse to illuminate the dielectric object. In order to reduce the number of the unknown parameters for the imaging problem, the shape function of the object is interpolated in terms of the cubic spline. The inverse problem is resolved by an optimization approach, and the global searching scheme APSO is then employed to search the parameter space. Numerical results demonstrate that, even when the initial guess is far away from the exact one, good reconstruction can be obtained.
\end{abstract}

\section{KEYWORDS}

FDTD, Multiple Dielectric Objects, Asynchronous Particle Swarm Optimization, Inverse Problems

\section{INTRODUCTION}

Microwave imaging is a kind of inverse scattering technique to estimate unknown objects. The scattered wave from an object carries information of electromagnetic properties of the scatterer, such as geometry, size, location and permittivity. The original object properties can be reconstructed by numerically time reversing the scattering process. Since there are many applications such as geophysical prospecting, medical imaging, non-destructive evaluated, and determination of underground tunnels, etc [1]-[3].

A variety of electromagnetic imaging techniques [4-6] have been proposed based on the finitedifference time-domain (FDTD) method to calculate inverse scattering problems. The nonlinearity of the problem is coped with by applying iterative optimization techniques [4]-[5].

Natarajan Meghanathan et al. (Eds) : ACSIT, FCST, ITCA, CoNeCo - 2015

pp. 35-42, 2014. ( ) CS \& IT-CSCP 2015

DOI : $10.5121 /$ csit.2015.51205 
Traditional iterative inverse algorithms are founded on a functional minimization via some gradient-type scheme. In general, during the search of the global minimum, they tend to get trapped in local minima when the initial guess is far from the exact one. In contrast to traditional deterministic methods, stochastic searching schemes, such as such as genetic algorithm[6], particle swarm optimization[7], provides a more robust and efficient approach for solving inverse scattering problems.

The particle swarm optimization (PSO) is a kind of evolutionary algorithm than has gained popularity in electromagnetic problem recently. One of the PSO advantage is the fact the very few parameters have to be adjusted to obtain the optimum results. In the recent, a new updating strategy for the PSO to produce the results with better performance than the original PSO, which named asynchronous particle swarm optimization (APSO)[8]. To the best of our knowledge, there is still no investigation on using the APSO to reconstruct the electromagnetic imaging of multiple dielectric objects under time domain. Thus, this paper presents a computational scheme combining the FDTD and APSO to reconstruct the microwave imaging of a 2D multiple dielectric objects $r$ with arbitrary cross section in free space.

\section{FORWARD PROBLEM}

Consider a homogeneous dielectric cylinder located in free space as depicted in Fig. 1. The cross section of the object is star like shape that can be representation in polar coordinates in the $x-y$ plane with respect to the center position. The permittivity and permeability of free space and dielectric object are denoted by and, respectively. The dielectric object is illuminated by Gaussian pulse line source located at the points denote by Tx and scattered waves are recorded at those points denoted by Rx. The computational domain is discretized by the Yee's cell. It should be mentioned that the computational domain is surrounded by the optimized PML absorber [9] to reduce the reflection from the air-PML interface.

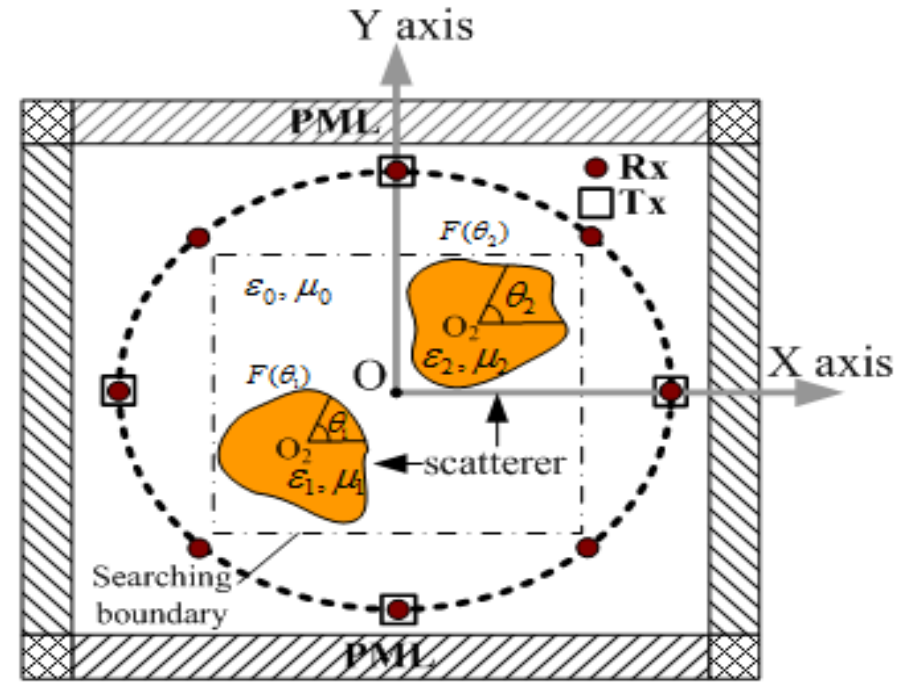

Figure 1. Geometrical configuration of the problem.

The direct scattering problem is to calculate the scattered electricfields while the shape, location and permittivity of the scatterer is given. The shape function $F(\theta)$ of the scatter is approximated by the trigonometric series in the direct scattering problem 


$$
F(\theta)=\sum_{n=0}^{N / 2} B_{n} \cos (n \theta)+\sum_{n=1}^{N / 2} C_{n} \sin (n \theta)
$$

where $B_{n}$ and $C_{n}$ are real coefficients to expand the shape function. In order to closely describe the shape of the cylinder for the forward scattering procedure, the sub gridding technique[10] is implemented in the FDTD code. For the time domain scattering and/or inverse scattering problem, the scatterers can be assigned with the fine region such that the fine structure can be easily described. If higher resolution is needed, only the fine region needs to be rescaled using a higher ratio for subgridding. This can avoid gridding the whole problem space using the finest resolution such that the computational resources are utilized in a more efficient way, which is quite important for the computational intensive inverse scattering problems.

\section{INVERSE PROBLEM}

For the inverse scattering problem, the shape, location and permittivity of the dielectric cylinder are reconstructed through the given scattered electric fields obtained at the receivers. This problem is formulated into an optimization approach, for which the global searching scheme APSO is employed to minimize the following cost function $(\mathrm{CF})$ :

$$
C F=\frac{\sum_{n=1}^{N_{i}} \sum_{m=1}^{M} \sum_{t=0 \Delta t}^{T}\left|E_{z}^{\exp }(n, m, t)-E_{z}^{c a l}(n, m, t)\right|}{\sum_{n=1}^{N_{i}} \sum_{m=1}^{M} \sum_{t=0 \Delta t}^{T}\left|E_{z}^{\exp }(n, m, t)\right|}
$$

where $E_{z}^{\exp }$ and $E_{z}^{c a l}$ are the experimental electric fields and calculated electric fields, respectively. The $\mathrm{N}_{\mathrm{i}}$ and $\mathrm{M}$ are the total number of the transmitters and receivers, respectively. T is the total time step number of the recorded electric fields.

\subsection{Asynchronous Particle Swarm Optimization (APSO)}

Particle swarm global optimization is a class of derivative-free, population-based and selfadaptive search optimization technique. Particles (potential solutions) are distributed throughout the searching space and their positions and velocities are modified based on social behavior. The social behavior in PSO is a population of particles moving towards the most promising region of the search space. Clerc [11] proposed the constriction factor to adjust the velocity of the particle for obtaining the better convergence; the algorithm was named as constriction factor method. PSO starts with an initial population of potential solutions that is randomly generated and composed of $N_{p}$ individuals (also called particles) which represents the permittivity, location and the geometrical radiuses of the objects.

After the initialization step, each particle of population has assigned a randomized velocity and position. Thus, each particle has a position and velocity vector, and moves through the problem space. In each generation, the particle changes its velocity by its best experience, called $x_{\text {pbest }}$, and that of the best particle in the swarm, called $x_{g b e s t}$. 
Assume there are $N_{p}$ particles in the swarm that is in a search space in $D$ dimensions, the position and velocity could be determine according to the following equations (constriction factor method):

$$
\begin{aligned}
& v_{i d}^{k}=\chi \cdot\left(v_{i d}^{k-1}+c_{1} \cdot \phi_{1} \cdot\left(x_{p b e s t, i d}-x_{i d}^{k-1}\right)+c_{2} \cdot \phi_{2} \cdot\left(x_{g b e s t, d}-x_{i d}^{k-1}\right)\right) \\
& x_{i d}^{k}=x_{i d}^{k-1}+v_{i d}^{k}
\end{aligned}
$$

where $\quad \frac{2}{x=\frac{2-\phi-\sqrt{\phi^{2}-4 \phi} \mid}{|c|}}, \phi=c_{1}+c_{2} \geq 4 \cdot c_{1}$ and $c_{2}$ are learning coefficients, used to control the impact of the local and global component in velocity equation (3). $v_{\mathrm{id}}^{\mathrm{k}}$ and $\mathrm{x}_{\mathrm{id}}^{\mathrm{k}}$ are the velocity and position of the $i$-th particle in the $d$-th dimension at $k$-th generation, $\varphi_{1}$ and $\varphi_{2}$ are both the random number between 0 and 1 .

The key distinction between APSO and a typical synchronous PSO is on the population updating mechanism. In the synchronous PSO, the algorithm updates all the particles velocities and positions using equations (3) and (4) at end of the generation, and then update the best positions, $x_{p b e s t}$ and $x_{\text {gbest }}$. Alternatively, the updating mechanism of APSO is that the new best position is found after each particle position updates if the best position is better than the current best position. The new best position will be used in following particles swarm immediately. The swarm reacts more quickly to speed up the convergence because the updating occurs immediately after objective function evaluation for each particle. The pseudo code of the APSO is listed as Table 1.

\begin{tabular}{|c|c|}
\hline \multicolumn{2}{|r|}{ pseudo code of the APSO. } \\
\hline 1. & randomly initialize the particles position and velocity; \\
\hline 2. & while The stoppingcriterion(number of iterations) doesn't meet do \\
\hline 3. & Evaluate the fitness (calculate the cost function) of each particle. \\
\hline 4. & fori $=1$ to $N_{p}$ (number of particles) do \\
\hline 5. & for $\mathrm{D}=1$ to maximum dimension do \\
\hline 6 & if The Fitness $\left(\mathrm{x}_{\mathrm{i}}\right)>$ Fitness $\left(\right.$ pbest $\left._{\mathrm{i}}\right)$ then \\
\hline 7. & pbest $_{\mathrm{i}}=\mathrm{x}_{\mathrm{i}}$. \\
\hline 8. & end if \\
\hline 9. & if Fitness $\left(\right.$ pbest $\left._{\mathrm{i}}\right)>$ Fitness $($ gbest $)$ then \\
\hline 10. & gbest $=$ pbest $_{\mathrm{i}}$ \\
\hline 11. & end if \\
\hline 12. & update particle's velocity and position usingequations 3 to 4 \\
\hline 13. & end for \\
\hline 14. & go to next iternation until meet stopping criterion. \\
\hline 15. & end while \\
\hline 16. & Return the position of gbest (the optimal filter mask). \\
\hline
\end{tabular}

Table 1.pseudo code of the APSO. 


\subsection{Cubic spline interpolation method}

In order to reduce the unknowns required to describe a cylinder of arbitrary cross section, the shape function of the cylinder is expressed in terms of a cubic spline. As shown in Figure 2, the cubic spline consists of the polynomials of degree 3. $P_{i}(\theta), i=1,2, \Lambda, N$. Through the interpolation of the cubic spline, an arbitrary smooth cylinder can be easily described through the radius parameters $\rho_{1}, \rho_{2}, \Lambda, \rho_{N}$ and the slope $\rho_{N}^{\prime}$

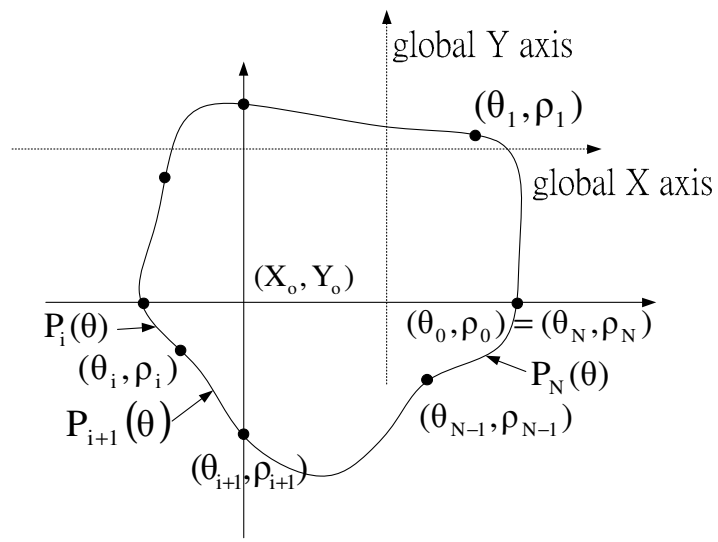

Figure 2. A cylinder with arbitrary shape is described in terms of the cubic spline.

\subsection{Numerical Results}

As shown in Figure 1, the problem space is divided in $100 \times 100$ grid cells with grid size $\Delta x=\Delta y$ $=5.95 \mathrm{~mm}$. The homogeneous dielectric cylinder is located in free space and illuminated by transmitters at four different positions $\left(N_{i}=4\right)$. The scattered $\mathrm{E}$ fields for each illumination are collected by eight receivers $(M=8)$ that are uniformly distributed along a circle. The transmitters and receivers are collocated at a distance of 40 grids from the origin. The excitation waveform $I_{z}(t)$ of the transmitter is the Gaussian pulse, given by:

$$
I_{z}(t)=\left\{\begin{array}{cr}
A e^{-\alpha(t-\beta \Delta t)^{2}}, & t \leq T_{w} \\
0, & t>T_{w}
\end{array}\right.
$$

where $\beta=24, A=1000, \Delta t=13.337 p s, T_{w}=2 \beta \Delta t$, and $\alpha=\left(\frac{1}{4 \beta \Delta t}\right)^{2}$.

The time durationis set to $350 \Delta t(K=350)$. Note that in order to describe the shape of the cylinder more accurately, the subgridding FDTD technique is employed both in the forward scattering (1:9) and the inverse scattering (1:5) parts - but with different scaling ratios as indicated in the parentheses. For the forward scattering, the E fields generated by the FDTD with finer subgrids are used to mimic the experimental data in (2).let us consider the problem for two separate dielectric cylinders o different relative permittivities. The first dielectric cylinder is located at $(-59.5 \mathrm{~mm},-35.7 \mathrm{~mm})$ of which the shape function is $F_{1}\left(\theta_{1}\right)=29.75+11.9 \cos \left(\theta_{2}\right) \mathrm{mm}$ 
and relative permittivity is $\varepsilon_{r, 1}=3.6$. The shape function and relative permittivity of the second dielectric cylinder are chosen as: $F_{2}\left(\theta_{2}\right)=29.75+5.95 \cos \left(\theta_{4}\right) \mathrm{mm}$ and $\varepsilon_{r, 2}=2.56$, respectively. The position of the other dielectric cylinder is $(35.7 \mathrm{~mm}, 35.7 \mathrm{~mm})$ ). The reconstructed images at different generations and the relative error of the final example are shown in Fig.3and Fig 4, respectively.

Figure 3 shows that the reconstructed image of the second object is better than the image of the first object. This is due to the fact that the intrinsic high scattering strength of the first object with higher dielectric constant is strong than the scattering strength of the second one. Thus, the minor relative errors of the reconstructed image of the strong scatterer have significant effect in reconstructed quality of the weak scatterer consequentially. The achieved shape error (DF) and relative permittivity error (DIPE) of the first object (strong scatterer) in the final generation are $1.4 \%$ and $0.4 \%$, respectively. The error of the relative permittivity (DIPE) of the second object (weak scatterer) is about $0.7 \%$ such that the shape error (DF) is raised to $4.2 \%$. Although the reconstructed quality of the weak scatterer is poorer than the quality of the strong scatterer, the proposed method still yield acceptable reconstructed results.



Figure 3.The reconstructed cross section of the cylinder at different generations.

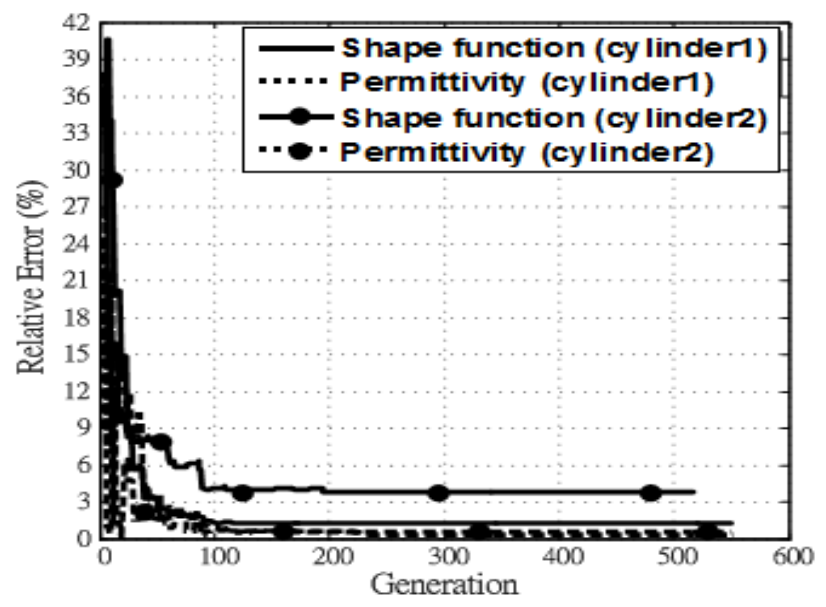

Figure 4.Shape function error and permittivity error at sequential generations. 


\section{CONCLUSIONS}

In this paper, we study the imaging reconstruction problem of multiple dielectric objects with arbitrary cross section in time domain By combining the FDTD method and the APSO, good reconstructed results are obtained. In order to describe the shape of the scatterer more effectively, a cubic spline interpolation technique is utilized. The inverse problem is reformulated into an optimization one, and then the global searching scheme APSO is employed to search the parameter space. By using the APSO, the shape, location and permittivity of the object can be successfully reconstructed. Numerical results have been carried out, even when the initial guess is far from the exact one, the APSO can still yield a good solution for the properties of the objects.

\section{ACKNOWLEDGEMENTS}

This work was supported by Ministry of Science and Technology,Republic of China, under grant number MOST 103-2221-E-229 -001.

\section{REFERENCES}

[1] F. Soldovieri, R. Solimene, A. Brancaccio, and R. Pierri, (2007)"Localization of the interfaces of a slab hidden behind a wall," IEEE Transactions on Geoscience and Remote Sensing, Vol. 45, No. 8, pp. 2471-2482.

[2] Y. Wang and A. E. Fathy, (2012)“Advanced System Level Simulation Platform for ThreeDimensional UWB Through-Wall Image" IEEE Transactions on Geoscience and Remote Sensing, Vol. 50, pp. 1986-2000.

[3] S. C. Hagness, A. T. Taflove, and J. E. Bridges, (1998) "Two dimension FDTD Analysis of a Pulsed Microwave Confocal System for Breast Cancer Detection: Fixed-Focus and Antenna Array Sensors" IEEE Transactions on Biomedical Engineering, Vol.45.

[4] I. T. Rekanos, (2003)“Time-domain inverse scattering using lagrange multipliers: an iterative FDTDbased optimization technique, "Journal of Electromagnetic Waves and Applications, vol. 17, No. 2, pp. 271-289,.

[5] T. Takenaka, H. Jia, and T. Tanaka, (2000)“Microwave imaging of electrical property distributions by a forward-backward time-stepping method," Journal of Electromagnetic Waves Application, vol. 14, pp. 1609-1625.

[6] C. H. Huang, C. C. Chiu, C. L. Li, and Y. H. Li, (2008)"Image Reconstruction of the Buried Metallic Cylinder Using FDTD Method and SSGA,” Progress In Electromagnetics Research, PIER 85, 195210.

[7] M. Donelli and A. Massa, (2005) "Computational approach based on a particle swarm optimizer for microwave imaging of two-dimensional dielectric scatterers," IEEE Transactions on Microwave Theory and Techniques, vol. 53, No. 5, pp. 1761 - 1776.

[8] A. Carlisle and G. Dozier, (2001)“An Off-The-Shelf PSO,” Proceedings of the 2001 Workshop on Particle Swarm Optimization, pp.1-6.

[9] C. L. Li, C. W. Liu, and S. H. Chen, (2003) "Optimization of a PML Absorber's Conductivity Profile using FDTD,” Microwave and Optical Technology Letters, vol. 37, pp. 380-383.

[10] M. W. Chevalier, R. J. Luebbers and V. P. Cable, (1997) "FDTD local grid with materical traverse," IEEE Trans. Antennas and Propagation, Vol. 45, No. 3,.

[11] M. Clerc, (1999) "The swarm and the queen: towards a deterministic and adaptive particle swarm optimization," Proceedings of Congress on Evolutionary Computation, Washington, DC, pp 19511957. 


\section{AUTHORS}

Chung-Hsin Huang was born in Tucheng, Taiwan, Republic of China, on February 1, 1980. He received M.S.E.E. and Ph.D degrees in electrical engineering from Tamkang University, Taipei, Taiwan, in 2004 and 2009 respectively. He is currently an Assistant Professor with the Department of Marine Engineering, Taipei College of Maritime Technology. His current research interests include inverse scattering problem, optimization methods, dielectric material characterization and wireless communications.

Chien-Hung Chen was born in Kaohsiung, Taiwan Republic of China, On March 8, 1971. He received Ph.D degree in electrical engineering form Tamkang University Taipei, Taiwan. He is currently an Assistant Professor with the Department of Information Technology and Mobile Communication. His current research interests include indoor wireless communications.

Jau-Je Wu was born in Taiwan, Republic of China. He receives the Ph.D degrees from Virginia Polytechnic Inst. and State Univ., Blacksburg University. In 2012 he joined the faculty of the Department of Marine Engineering, Taipei College of Maritime Technology, Taipei City, Taiwan, where he is now an Associate Professor. His current research interests include numerical techniques in Structural Mechanics.

Dar-Sun Liu was born in Taiwan, Republic of China. He receives the Ph.D degrees from National University, Taiwan. In 2012 he joined the faculty of the Department of Marine Engineering, Taipei College of Maritime Technology, Taipei City, Taiwan, where he is now an Associate Professor. His current research interests include Micro fluidic device and Modular design.
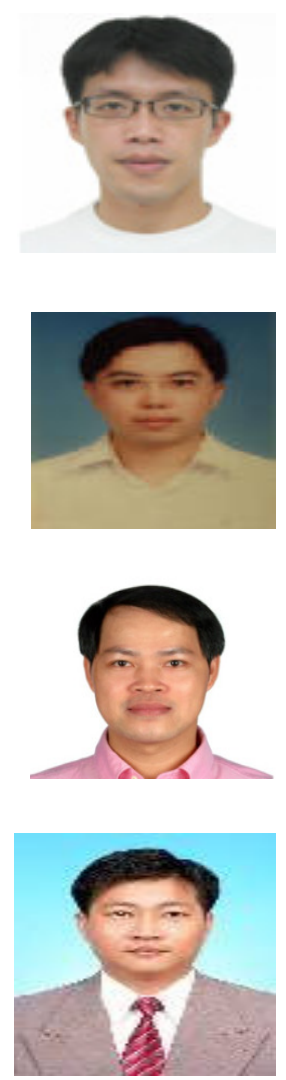